Claude Guerin

Frédérique Bayle

Véronique Leray

Sophie Debord

Alina Stoian

Hodane Yonis

Jean-Baptiste Roudaut

Gael Bourdin

Mojgan Devouassoux-Shisheboran

Elodie Bucher

Louis Ayzac

Sylvie Lantuejoul

Carole Philipponnet

Jean Louis Kemeny

Bertrand Souweine

Jean-Christophe Richard

\section{Erratum to: Open lung biopsy in nonresolving ARDS frequently identifies diffuse alveolar damage regardless of the severity stage and may have implications for patient management}

Published online: 16 January 2015

(C) Springer-Verlag Berlin Heidelberg and ESICM 2015

The online version of the original article can be found under doi:10.1007/s00134-014-3583-2.

C. Guerin $(\bowtie) \cdot$ F. Bayle $\cdot$ V. Leray $\cdot$ S. Debord ·

A. Stoian - H. Yonis - J.-B. Roudaut - G. Bourdin - J.-C. Richard Service de Réanimation Médicale, Hôpital de la Croix Rousse,

Hospices Civils de Lyon, Université de Lyon, 103 Grande Rue de la Croix-Rousse, 69004 Lyon, France

e-mail: claude.guerin@chu-lyon.fr

Tel.: 33(0)472071762

C. Guerin · J.-C. Richard

CREATIS INSERM 1044, Villeurbanne, France

M. Devouassoux-Shisheboran

Laboratoire d'Anatomie Pathologique,

Hôpital de la Croix Rousse, Hospices Civils de Lyon,

Université de Lyon, Lyon, France

E. Bucher

Département d'Information Médicale,

Hôpital de la Croix Rousse, Hospices Civils de Lyon, Lyon, France

L. Ayzac

Centre de coordination et de lutte contre les infections

nosocomiales, Hôpital Henri Gabrielle, Hospices Civils de Lyon,

Saint-Genis Laval, France
S. Lantuejoul

Département d'Anatomie et Cytologie Pathologiques DACP, Pôle de Biologie, Institut de Biologie et de Pathologie,

CHU A Michallon, Grenoble, France

C. Philipponnet · B. Souweine

Service de Réanimation Médicale, Hôpital Gabriel Monpied, CHU de Clermont-Ferrand, Clermont-Ferrand, France

J. L. Kemeny

Service d'anatomie pathologique, CHU de Clermont-Ferrand,

Clermont-Ferrand, France

\section{Erratum to: Intensive Care Med} DOI 10.1007/s00134-014-3583-2

Table 2 incorrectly states that 22 of the 30 non-ARDS patients showed diffuse alveolar damage on open lung biopsy. The correct number is $8 / 30$. 